\title{
NECROTIZING LYMPHADENITIS AS A CAUSE OF PERIPHERAL LYMPHADENOPATHY IN A PATIENT WITH SYSTEMIC LUPUS ERYTHEMATOSUS: A CASE REPORT
}

\author{
Vanessa Posener de Andrade ${ }^{1}$, Jucier Gonçalves Júnior ${ }^{1, \star}$, Victor Caires Tadeu ${ }^{1}$, Sandra Gofinet Pasoto ${ }^{1}$ \\ 1.Universidade de São Paulo, São Paulo (SP), Brazil. \\ ${ }^{\star}$ Corresponding author: juciergjunior@hotmail.com
}

\section{BACKGROUND}

Necrotizing lymphadenitis (NL) as an isolated manifestation of systemic lupus erythematosus (SLE) is a rare condition, especially in patients over 50 years of age, and must be differentiated from other causes of lymph node enlargement. We aimed to report a case of an adult patient with SLE and NL who had no additional active organ involvement.

\section{CASE REPORT}

A 59-year-old white female had been diagnosed with SLE 25 years before, with cutaneous (photosensitivity), articular, hematologic (lymphopenia and thrombocytopenia), and pulmonary (bronchiolitis) involvement. She had achieved disease remission 2 years earlier, with no current immunosuppressive therapy or hydroxychloroquine (because of preexisting maculopathy). She then presented with a 3-month history of bilateral enlarged cervical lymph nodes, which continued to increase in size, as well as night sweats, asthenia, and fatigue. The workup included negative anti-dsDNA antibodies, C3 and C4 levels of 135 and 13.3, respectively, and negative serologies (hepatitis, Epstein-Barr, cytomegalovirus, toxoplasma, and rubella). Her neck computed tomography (CT) scan showed bilateral enlarged lymph nodes, some of them globose and contrast-enhancing, with the largest one measuring $2.3 \mathrm{~cm}$. Chest, abdomen, and pelvis CT scanning showed no abnormalities. Fine-needle aspiration (FNA) of the largest lymph node for cytology was performed and demonstrated reactive lymphadenitis with a negative acid-fast bacilli (AFB) smear and negative bacterial and fungal cultures. An excisional biopsy of a left cervical lymph node was then carried out and uncovered reactive follicular lymphoid hyperplasia associated with NL. Immunohistochemistry showed mixed populations of lymphocytes (BCD20+ / TCD3+), no AFB, fungi, or signs of malignancy; biopsy specimen cultures were also negative. The diagnosis of SLE-associated NL was thus considered. The patient was then started on azathioprine $(1.5 \mathrm{mg} / \mathrm{kg} /$ day $)$ and prednisone $(5 \mathrm{mg} / \mathrm{day})$ for her constitutional symptoms and increasing lymph nodes, in view of the contraindication to hydroxychloroquine. In addition, a follow-up appointment was scheduled.

\section{CONCLUSION}

Necrotizing lymphadenitis, even if presenting as the sole organ involvement, should be considered in the differential diagnosis of peripheral lymphadenopathy in patients previously diagnosed with SLE.

\section{KEYWORDS}

Case report, Necrotizing lymphadenitis, Lymphadenitis, Systemic lupus erythematosus. 